\title{
A NEURO-FUZZY INFERENCE MODEL FOR BREAST CANCER RECOGNITION
}

\author{
Bekaddour Fatima $^{1}$ and Chikh Mohammed Amine ${ }^{2}$ \\ ${ }^{1}$ Departement of Computer Science, Abou Bekr Belkaid University, Tlemcen, Algeria \\ fatima.bekaddouregmail.com \\ ${ }^{2}$ Departement of Computer Science, Abou Bekr Belkaid University, Tlemcen, Algeria \\ am_chikh@yahoo.fr
}

\begin{abstract}
Breast cancer is known as one of the most common cancers to afflict the female population. Computer assisted diagnosis can be helpful for doctors in detection and diagnosing of potential abnormalities. Several techniques can be useful for accomplishing this task. This paper outlines an approach for recognizing breast cancer diagnosis using neuro-fuzzy inference technique namely ANFIS (Adaptative Neuro-Fuzzy Inference System). Wisconsin breast cancer diagnosis (WBCD)database developed at University of California, Irvine (UCI) is used to evaluate this method. Results show that the best performances are obtained by our model compared to others cited in literatur (an accuracy of 98, $25 \%$ ).
\end{abstract}

\section{KEYWORDS}

Artificial Neural Networks, Breast Cancer, fuzzy logic, neuro-fuzzy, computer assisted diagnostic WBCD, Artificial Intelligence

\section{INTRODUCTION}

In [5] , breast cancer has been one of the major causes of death among women and a true emergency for the healthcare systems of industrialized countries. It is the second leading cause of cancer deaths among women in the world [2].It is characterized by an abnormal multiplication of a cell in the human body. Not entailing serious consequences, early, cancer can be developed into a serious condition if treatment is not done on time. Due to its late diagnosis, it often causes a mutilating and expensive treatments accompanied by a high mortality rate. It has the form of lumps or tumors in the tissues of the breast. Tumors can either be malignant or benign. However, differentiating a malignant tumor from a benign one is a very tedious task due to the structural similarities between the two (figure 1) [24]

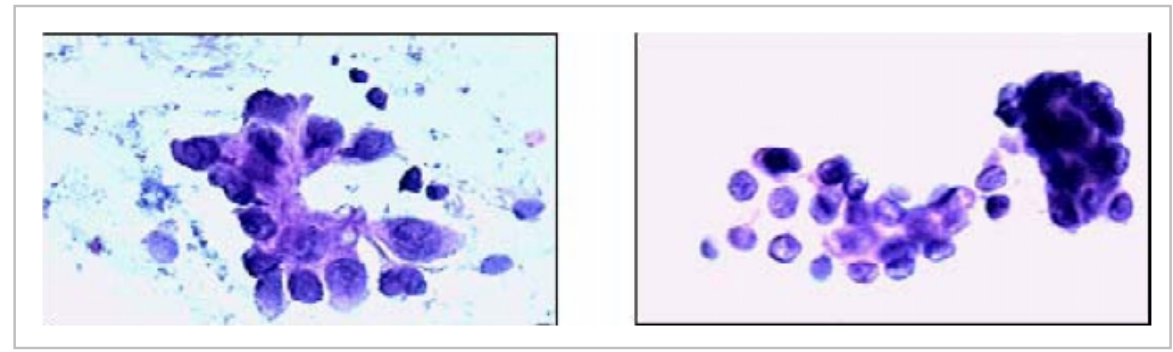

Figure 1: Fine Needle Biopsies of Breast. Malignant (left) And benign (right) breast tumors [33]

DOI : $10.5121 /$ ijcsit.2012.4513 
It is an extremely critical and time consuming task for the physician to accurately identify the structural differences.The question remains if there could be an automated technique that could relieve the physician of the tedious task of distinguishing a malignant tumor from a benign one [24]. Computer aided diagnosis systems are important for pattern recognition, aiming to assist doctors in making diagnosis decisions. Machine learning have been successfully applied in computer-aided diagnosis (CAD) systems [7], [34], [25]. These methods learn hypotheses from a large amount of diagnosed samples, i.e., the data collected from a number of necessary medical examinations along with the corresponding diagnoses made by medical experts, to assist the medical experts in making a diagnosis in the future [13]

In the literature, several methods have been applied to detect the presence of cancer in the breast. Most work reported employs Neural Networks , Genetic Algorithm (GA), Fuzzy Inference System (FIS) and Neuro-Fuzzy Hybrid Models for breast cancer classification. Some literature works based on WBCD (Wisconsin breast cancer diagnosis) are shown in the following Table1:

TABLE 1: Some Literature Works

\begin{tabular}{|c|c|c|}
\hline References & Technique & $\begin{array}{c}\text { Classification rate } \\
(\mathbf{\%})\end{array}$ \\
\hline$[3]$ & IGANFIS & 98.24 \\
\hline$[32]$ & SANFIS & 96.07 \\
\hline$[10]$ & L.V .Q & 95,82 \\
\hline$[15]$ & Fuzzy & 96,71 \\
\hline$[21]$ & Fuzzy-GA1 & 97,36 \\
\hline
\end{tabular}

Neuro-Fuzzy Hybrid models improve relatively remarkable performances in diagnosis [28] . There exists many kinds of neuro-fuzzy classifiers such as trainable fuzzy classification systems [20][8], histogram based fuzzy systems [4][14] ,fuzzy sets self organizing classification systems [12][11][16], Neuro-fuzzy classification systems (NeFClass)[27]...

This paper presents the Adaptive Neuro-Fuzzy Inference System (ANFIS), which is well-suited to classification of qualitative input and output variables. The rest of the paper is organized as follows. First, we introduce artificial neural network (ANN) and the neuro-fuzzy approach .Then, the dataset used for breast cancer diagnosis is described in the second section. The final section views and analyzes results obtained for classification of the breast cancer probelm.

\section{NEURO-FUZZY SYSTEMS}

Neural networks and fuzzy logic are two approaches that are widely used to solve classification and pattern recognition problems. The main advantage of neural networks is their learning capabilities and their ease of implementation. In the other hand , the non interpretability of their results is a major disadvantage (black boxes). The fuzzy inference systems can interpret their results using a knowledge base (rule base). The joint use of neural networks and fuzzy inference systems can exploit the advantages of both methods. 
International Journal of Computer Science \& Information Technology (IJCSIT) Vol 4, No 5, October 2012

Table 2 below gives a comparative view between the two approaches:

TABLE 2: Comparison between neural networks and fuzzy inference systems [18]

\begin{tabular}{|c|c|}
\hline Artificial neural network & Fuzzy inference system \\
\hline $\begin{array}{c}\text { Difficult to use prior rule } \\
\text { knowledge }\end{array}$ & $\begin{array}{c}\text { prior rule base can be } \\
\text { incorporated }\end{array}$ \\
\hline Based on Learning & Can not learn \\
\hline Black box & Interpretable(If - Then rules) \\
\hline $\begin{array}{c}\text { Complicated learning } \\
\text { algorithms }\end{array}$ & $\begin{array}{c}\text { Simple interpretation and } \\
\text { implementation }\end{array}$ \\
\hline Difficult to extract knowledge & Knowledge can be available \\
\hline
\end{tabular}

We can say that neuro-fuzzy systems are connectionist models that allow learning as artificial neural network, but their structure can be interpreted as a set of fuzzy rules .Fuzzy logic and neural networks form the basis of the majority aided diagnostic intelligent systems.It would be interesting to combine the two approaches to exploit both advantages.

Different models exist for combining fuzzy logic and neural networks. In this paper we diagnose breast cancer using the ANFIS approach (Adaptive Neuro Fuzzy Inference System) proposed by Jang [9].

\subsection{ANFIS Architecture}

The Anfis (acronym of Adaptative Neuro Fuzzy Inference System) is a neuro-fuzzy model proposed by Jang in [9] . Jang combined both Fuzzy Logic and Neural Network to produce a powerful processing tool named Neuro-Fuzzy Systems that have both Neural Network and Fuzzy Logic advantages and the most common one is ANFIS .Actually, this tool is like a fuzzy inference system, but the difference is in the use of a back propagation algorithm for minimizing the error [29].The Anfis architecture is illustrated in the figure 2:

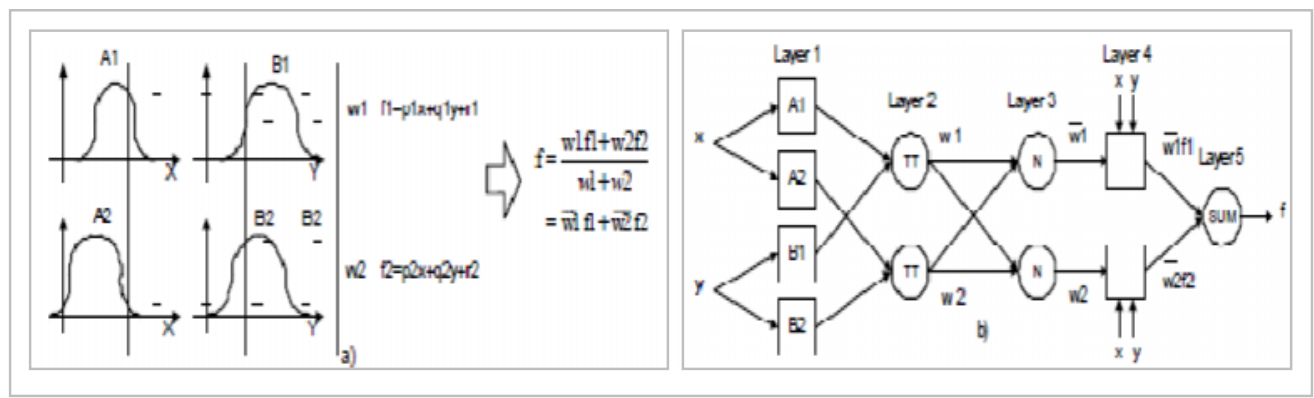

Figure 2. a) A two input first-order

b) Equivalent Anfis Architecture Sugeno fuzzy model with two rules

For simplicity, we assume that the fuzzy inference system under consideration has two inputs $\mathrm{x}$ and $y$ and one output $\mathrm{z}$.for a first-order Sugeno fuzzy model, a common rule set with two fuzzy if-then rules is the following:

R1: If $x$ is $A 1$ and $y$ is $B 1$ then $f 1=p 1 x+q 1 y+r 1$

R2: If $x$ is $A 2$ and $y$ is $B 2$ then $f 2=p 2 x+q 2 y+r 2$ 
International Journal of Computer Science \& Information Technology (IJCSIT) Vol 4, No 5, October 2012

Figure 2.a) gives an illustration about the reasoning mechanism for this Takagi-Sugeno Model. Figure 2.b) shows the corresponding equivalent ANFIS architecture where nodes of the same layer have similar functions.

As illustrate figure 2.b), the Anfis architecture contains fixed nodes (circular) and adaptive nodes (square) that allows representing the basic rules carried out by an adaptative network .

Note that the structure of this adaptative network is not unique, we can combine layer 3 and 4 to obtain an equivalent network with only four layers .The first hidden layer is for fuzzification of the input variables and T-norm operators are deployed in the second hidden layer to compute the rule antecedent part. The third layer normalizes the rule strengths and the consequent parameters of the rules are determined in the fourth layer [1]. Output layer computes the overall input as the summation of all incoming signals [1]

\subsection{Hybrid learning algorithm}

Table 3 shows how to apply the hybrid learning algorithms to indentify Anfis parameters. The learning algorithm is composed of two phases:

- In the forward pass of the hybrid learning algorithm, node outputs values go forward until layer 4 and the consequent parameters are identified by the least squares method [9]

- In the backward pass, the premise parameters are adjusted using the gradient descent method [9].

TABLE 3. Two passes in the hybrid learning procedure for ANFIS [18]

\begin{tabular}{|l|l|l|}
\hline & Forward Pass & Backward Pass \\
\hline Premise Parameters & Fixed & Gradient Descent \\
\hline Consequent Parameters & Least-squares estimator & Fixed \\
\hline
\end{tabular}

The output ' $\mathrm{f}$ ' in Figure $\mathbf{2}$ b) can be written as:

$$
\begin{aligned}
f & =\frac{w 1}{w 1+w 2} f 1+\frac{w 2}{w 1+w 2} f 2 \\
& =\overline{w 1}(p 1 x+q 1 y+r 1)+\overline{w 2}(p 2 x+q 2 y+r 2) \\
& =(\overline{w 1} x) p 1+(\overline{w 1} y) q 1+(\overline{w 1}) r 1+(\overline{w 2} x) p 2+(\overline{w 2} y) q 2+(\overline{w 2}) r 2
\end{aligned}
$$

This way an adaptive network that is functionally equivalent to a first order Sugeno fuzzy model is constructed. [31]

\section{DADASET DESCRIPTION}

This section describes the database used for medical diagnosis problem. In this study we used the dataset provided by researchers at the University of Wisconsin. The dataset was obtained from the University of California Irvine (UCI) Machine Learning Repository [19] consist of 699 data with $65.5 \%$ classified as benign and $34.5 \%$ as malignant The Wisconsin breast cancer diagnosis (WBCD) database is the effort made at the University of Wisconsin Hospital for accurately diagnosing breast masses based solely on an FNA (Fine Needle Aspirates) test [6][21] .Nine visually assessed charac-teristics of an FNA sample considered relevant for diagnosis were identified, and assigned an integer value between 1 and 10[26].The measured variables are described in Table 4.

TABLE 4. Attributes of the diagnostic base 


\begin{tabular}{|c|c|c|c|}
\hline Configuration & $\begin{array}{c}\text { Number of } \\
\text { rules }\end{array}$ & $\begin{array}{c}\text { Learning } \\
\text { Error }\end{array}$ & $\begin{array}{c}\text { Classification } \\
\text { rate \% }\end{array}$ \\
\hline $2 * 2 * 2 * 2 * 2 * 2$ & 64 & 0.29843 & 0.9825 \\
\hline $2 * 3 * 3 * 3 * 2 * 2$ & 216 & 0.16176 & 0.8904 \\
\hline $2 * 2 * 2 * 3 * 2 * 3$ & 144 & 0.16982 & 0.9386 \\
\hline
\end{tabular}

The Wisconsin Breast Cancer Diagnosis (WBCD) problem involves classifying a presented case as to whether it is benign or malignant [22],[30].There are several studies based on this database. Among them, researchers having interpretability of the diagnostic as a prior objective have applied the method of extracting boolean rules from neural networks [22],[23]. Our work for the WBCD problem showed that it is possible to obtain diagnostic systems exhibiting high performance, coupled with interpretability and a confidence measure. The database itself consists of 699 cases including 16 unavailable instances as shown in Table 5

TABLE 5 . The Wisconsin Breast Cancer Data base

\begin{tabular}{|l|l|l|l|l|l|l|l|l|l|l|}
\hline Case & CT & UCS & UCH & MA & SEC & BN & BC & NN & M & Diagnosis \\
\hline 1 & 5 & 1 & 1 & 1 & 2 & 1 & 3 & 1 & 1 & Benign \\
\hline 2 & 5 & 4 & 4 & 5 & 7 & 10 & 3 & 2 & 1 & Benign \\
\hline$\cdots$ & $\cdots$ & $\cdots$ & $\cdots$ & $\cdots$ & $\cdots$ & $\cdots$ & $\cdots$ & $\cdots$ & $\cdots$ & $\cdots$ \\
\hline 683 & 4 & 8 & 8 & 5 & 4 & 5 & 10 & 4 & 1 & Malignant \\
\hline
\end{tabular}

\section{RESULTS AND DISCUSSION}

\subsection{Choice of Parameters}

we note that choice of the number of membership functions is important for the development of neuro-fuzzy systems. This affects the number of rules generated. Our goal is to obtain a high performance with a reasonable number of rules. In this study, we have used information gain algorithm [3] in order to reduce the feature number of the Wisconsin breast cancer database (WBCD), So we obtain 6 features instead of 9. Table 6 shows some results obtained for our experimentation:

TABLE 6. Error and classification rate for different configurations

\begin{tabular}{|c|c|}
\hline Attributes & Signification \\
\hline CT & Clump Thickness \\
\hline UCS & Uniformity of Cell Size \\
\hline UCH & Uniformity of Cell Shape \\
\hline MA & Marginal Adhesion \\
\hline SEC & Single Epithelial Cell Size \\
\hline BN & Bare Nuclei \\
\hline BC & Bland Chromatin \\
\hline NN & Normal Nucleoli \\
\hline M & Mitosis \\
\hline
\end{tabular}


We have assigned the values of error threshold to 0.001 . Besides, we adopt the TSK as the structure for the Fuzzy model. After several trials, we have chosen to assign two membership functions for each descriptor in order to reduce the size of rules generated (sm: small, bg: big). Regarding the type of membership functions, we chose generalized bell functions (to guard the readability of results).

Initial parameters of membership functions are shown in the following figure 3:

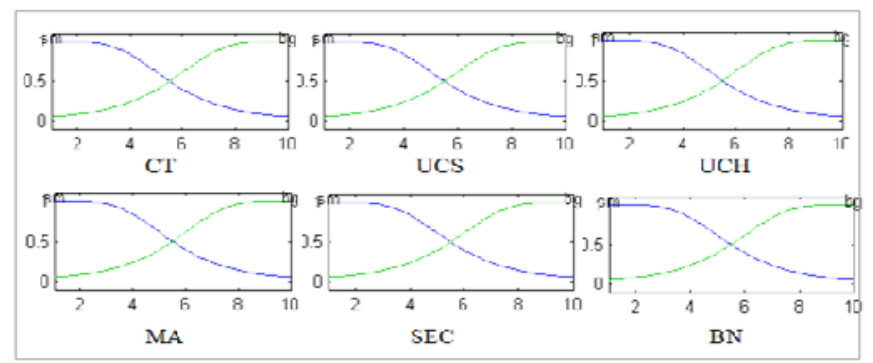

The structure of the proposed neuro-fuzzy model is presented in the following figure 4:

Figure 3. Initial membership functions (before learning)

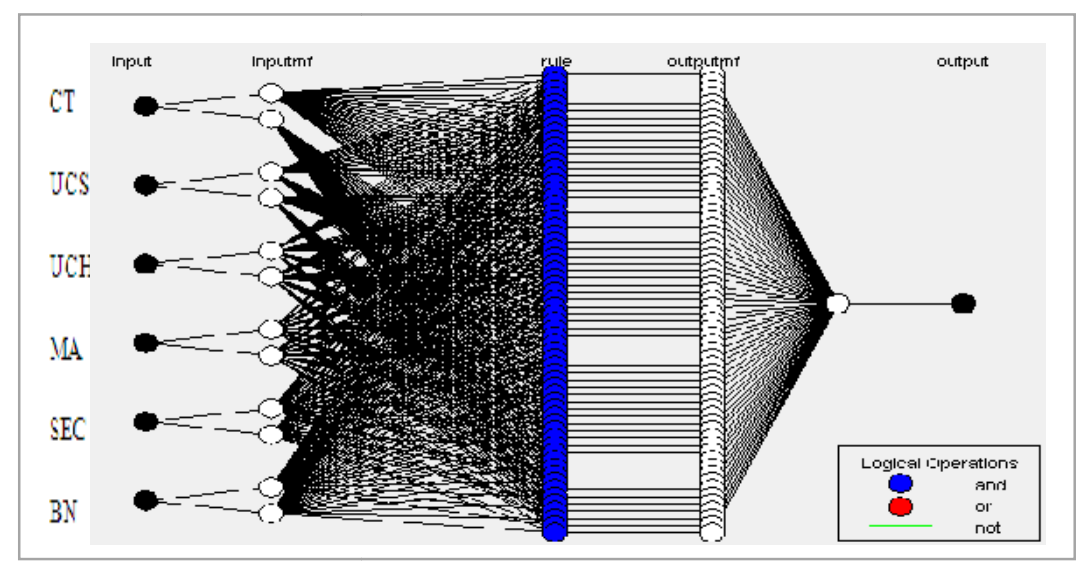

After choosing these initial configurations, we start learning phase, using the back propagation algorithm and the hybrid method (based on back propagation and least squares techniques).

\subsection{Generated Fuzzy rules}

At the end of learning, membership parameters will be modified as shown on figure 5:

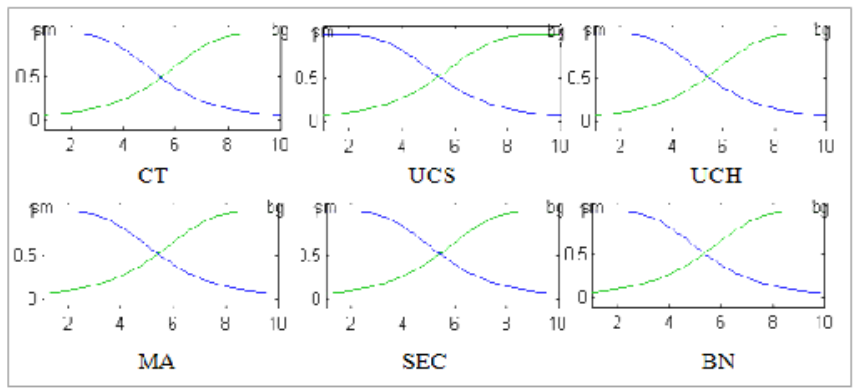

Figure 5. Final membership functions (after learning) 
International Journal of Computer Science \& Information Technology (IJCSIT) Vol 4, No 5, October 2012

Rules generated by our neuro-fuzzy model are divided into two groups:

- $\quad 1^{\text {st }}$ group : Fuzzy rules indicating benign class $(1,2,5,7,8,9,11,14,17,20,22,25,31,33,39,45$, $50,51,53,56)$.

- $\quad 2^{\text {nd }}$ group : Fuzzy rules indicating malignant class $(3,4,6,10,13,15,16,18,19,21,23,24,26$, $27,28,29,30,32,34,35,36,37,38,40,41,42,43, \quad 44,46,47,48,49,52,54,55,57, \quad 58,59,60, \quad 61,62$ $, 63,64)$

We coded the two outputs by two classes:

$*$ Benign Class $=0$ and $*$ Malignant class $=1$.

\subsection{Results Analysis}

The performances of the neuro-fuzzy classifier were evaluated using the following parameters:

1. $\mathrm{CC}=\mathrm{TP}+\mathrm{TN} /(\mathrm{TP}+\mathrm{TP}+\mathrm{FP}+\mathrm{FN}) * 100$ is the correct classification rate.

2. Error_rate $=\mathrm{FP}+\mathrm{FN} /(\mathrm{TP}+\mathrm{TP}+\mathrm{FP}+\mathrm{FN}) * 100$ is the error rate

3. Se : Sensitivity $=\mathrm{TP} /(\mathrm{TP}+\mathrm{FN}) * 100$ is the true positive rate.

4. Sp : Specificity $=\mathrm{TN} /(\mathrm{TN}+\mathrm{FP}) * 100$ is the fraction of nonevents that has been correctly rejected.

5. Nbr TP: is the number of True Positives.

6. Nbr TN: is the number of True Negatives.

7. Nbr FP: is the number of False Positives.

8. Nbr FN: is the number of False Negatives.

Performances of the neuro-fuzzy systems using hybrid and back-probagation algorithms are summarized in Table 7:

TABLE 7: Differentes results of Anfis

\begin{tabular}{|c|c|c|c|c|c|c|c|c|}
\hline $\begin{array}{c}\text { CC } \\
\mathbf{\%}\end{array}$ & $\begin{array}{c}\text { Error rate } \\
\mathbf{\%}\end{array}$ & $\begin{array}{c}\text { Se } \\
\mathbf{\%}\end{array}$ & $\begin{array}{c}\mathbf{S p} \\
\mathbf{\%}\end{array}$ & $\begin{array}{c}\mathbf{N b r} \\
\mathbf{T p}\end{array}$ & $\begin{array}{c}\mathbf{N b r} \\
\mathbf{T n}\end{array}$ & $\begin{array}{c}\mathbf{N b r} \\
\mathbf{F p}\end{array}$ & $\begin{array}{c}\mathbf{N b r} \\
\mathbf{F n}\end{array}$ & Type \\
\hline 98.25 & 1.75 & 97.5 & 98.65 & 78 & 146 & 2 & 2 & hybrid \\
\hline 64.91 & 35.09 & 0 & 100 & 76 & 148 & 0 & 80 & $\begin{array}{c}\text { Back- } \\
\text { propagation }\end{array}$ \\
\hline
\end{tabular}

We note that the hybrid method (which combine back-propagation and least squares for learning) gives better results than back propagation method, because the conclusion of the rules are adjusted using least square method.The confusion matrix showing the classification results of the ANFIS model is given in Table 8 : 
TABLE 8: Confusion Matrix

\begin{tabular}{|l|c|c|}
\hline $\begin{array}{c}\text { Desired \& Output } \\
\text { result }\end{array}$ & Benign records & Malignant records \\
\hline Benign records & 146 & 2 \\
\hline Malignant records & 2 & 78 \\
\hline
\end{tabular}

\subsubsection{Correctly recognized cases}

\subsubsection{Cancerous cases correctly recognized}

We calculated the degree of solicitation (degree of activation between 50\% and 100\%) for each rule in relation to the number of examples. The results are shown in the following Table 9 :

TABLE 9: Degree of solicitation for rules in T.P case

\begin{tabular}{|c|c|}
\hline Rule & Solicitation degree (\%) \\
\hline 64 & $8 / 80=10 \%$ \\
\hline 62 & $3 / 80=3 \%$ \\
\hline 63 & $4 / 80=5 \%$ \\
\hline 58 & $2 / 80=2,5 \%$ \\
\hline
\end{tabular}

Rules cited in Table 9 are presented below:

R58: If (CT is bg,UCS is $\mathbf{b g}, \mathrm{UCH}$ is $\mathbf{b g}, \mathrm{MA}$ is $\mathbf{s m}, \mathrm{SEC}$ is $\mathbf{s m}$ and $\mathrm{BN}$ is $\mathbf{b g}$ ) then Class $=1$ R62:If (CT is bg,UCS is bg,UCH is bg,MA is bg,SEC is $\mathbf{~} \mathbf{b m}$ and BN is $\mathbf{b g}$ ) then Class = 1 R63:If (CT is bg,UCS is bg, $\mathrm{UCH}$ is $\mathbf{b g}, \mathrm{MA}$ is bg, $\mathrm{SEC}$ is $\mathbf{b g}$ and $\mathrm{BN}$ is $\mathbf{s m}$ ) then Class $=1$ R64:If (CT is bg, UCS is $\mathbf{b g}, \mathrm{UCH}$ is $\mathbf{b g}, \mathrm{MA}$ is $\mathbf{b g}$, SEC is $\mathbf{b g}$ and $\mathrm{BN}$ is $\mathbf{b g}$ ) then Class = 1 We note that: Sm : Small, bg : big .

According to these results, Rule $\mathbf{6 4}$ has the most important degree of solicitation. We take two examples with a CT higher than 5, so the activated fuzzy rules and their degrees of solicitation are shown below:

\section{$1^{\text {st }}$ exemple :}

- $(\mathrm{CT}=8, \mathrm{UCS}=10, \mathrm{UCH}=10, \mathrm{MA}=7, \mathrm{SEC}=10, \mathrm{BN}=10)$ activates essentially the rule number $\mathbf{6 4}$ with a degree of solicitation equals to $82.25 \%$.

$2^{\text {nd }}$ example:

- $(\mathrm{CT}=6, \mathrm{UCS}=10, \mathrm{UCH}=10, \mathrm{MA}=10, \mathrm{SEC}=10, \mathrm{BN}=10)$ activates basically rules number $\mathbf{6 4}$ and 32 with respectively degree of solicitation equal to $62.11 \%$ and $37.71 \%$. Rule 32 is given below:

R32:If (CT is sm, UCS is $\mathbf{b g}, \mathrm{UCH}$ is $\mathbf{b g}, \mathrm{MA}$ is $\mathbf{b g}, \mathrm{SEC}$ is $\mathbf{b g}$ and $\mathrm{BN}$ is $\mathbf{b g}$ ) then Class $=1$. For the two previous examples the CT has gone beyond normal which makes the malignancy. 
International Journal of Computer Science \& Information Technology (IJCSIT) Vol 4, No 5, October 2012

\subsubsection{Non cancerous cases correctly recognized}

The degrees of solicitation for rules that represent the non-cancerous cases correctly recognized are presented in the Table 10:

TABLE 10: Degree of solicitation for rules in T.N case

\begin{tabular}{|c|c|}
\hline Rule & Solicitation degree (\%) \\
\hline 1 & $140 / 148=95 \%$ \\
\hline 33 & $3 / 148=2 \%$ \\
\hline
\end{tabular}

Rules presented in table 9 are:

R1: If (CT is $\mathbf{s m}, \mathrm{UCS}$ is $\mathbf{s m}, \mathrm{UCH}$ is $\mathbf{s m}, \mathrm{MA}$ is $\mathbf{s m}, \mathrm{SEC}$ is $\mathbf{s m}$ and BN is $\mathbf{s m}$ ) then Class $=0$. R33: If (CT is $\mathbf{b g}$, UCS is $\mathbf{s m}, \mathrm{UCH}$ is $\mathbf{s m}, \mathrm{MA}$ is $\mathbf{s m}, \mathrm{SEC}$ is $\mathbf{s m}$ and BN is $\mathbf{s m}$ ) then Class $=0$. We note that the first rule has the greatest solicitation degree.

If we take the first example in the diagnostic database having the following parameters:

- $(\mathrm{CT}=1, \mathrm{UCS}=1, \mathrm{UCH}=1, \mathrm{MA}=1, \mathrm{SEC}=2, \mathrm{BN}=4)$ has activated :

- The first rule (rule 1) with a degree of $81.40 \%$.

- The second rule (rule 2 ) with a degree of $2.72 \%$.Noting that Rule 2 says:

If (CT is $\mathbf{s m}, \mathrm{UCS}$ is $\mathbf{s m}, \mathrm{UCH}$ is $\mathbf{s m}, \mathrm{MA}$ is $\mathbf{s m}, \mathrm{SEC}$ is $\mathbf{s m}$ and $\mathrm{BN}$ is $\mathbf{b g}$ ) then Class $=0$

\subsection{2 . Misrecognized Cases:}

\subsubsection{Non cancerous cases predicted as cancerous (FP)}

Among the non-cancerous cases, our model has recognized two cases as cancerous, but only one example has a degree of solicitation between 50\% and 100\%. The Table 11 shows this case.

TABLE 11 : Degree of solicitation for rules in F.P case

\begin{tabular}{|c|c|}
\hline Rule: & Solicitation degree \%: \\
\hline 33 & $1 / 148=0.006$ \\
\hline
\end{tabular}

\subsubsection{Cancerous cases predicted as non cancerous $(\mathrm{FN})$}

We have just two examples where cancerous cases have been predicted as non cancerous with degree of solicitation less than $40 \%$ in most cases.

\section{CONCLUSION}

This work presents a knowledge extraction and classification of breast cancer disease using basically a neuro-fuzzy approach for system design, able to explain human decisions. We can say 
that the proposed method is an important tool which can be integrated in a CAD (computer aided diagnosis) for assisting in diagnostic decision making, with providing an understandable explanation of the underlying reasoning. According to results obtained in table $\mathbf{7 , 8}$ and others, we can say that the used method is very promising approach in medical data recognition .

\section{REFERENCES}

[1] A. Abraham ,"Adaptation of Fuzzy Inference System Using Neural Learning”,Computer Science Department, Oklahoma State University, USA ajith.abraham@ieee.org, http://ajith.softcomputing.net . pp : 60-62 . (2005).

[2] Mehmet Fatih Akay, "Support vector machines combined with feature selection for breast cancer diagnosis", Expert Systems with Applications 36; pp: 3240-3247,(2009).

[3] M. Ashraf, Kim Le , Xu Huang . "Information Gain and Adaptive Neuro-Fuzzy inference System for Breast Cancer Diagnoses', Computer Sciences and Convergence Information Technology (ICCIT), 5th International Conference on 2010, IEEE conference, pp : 911 - 915, (2010).

[4] Blackmore S., "Intelligent Sensing and Self-organizing Fuzzy-Logic Techniques used in Agricultural Automation", Poster Presentation, Asae/Csae Meeting Presentation, Silsoe College, Cranfield University. (1994).

[5] F.A. Cardillo, A. Starita, D. Caramella, A. Cilotti .A NEURAL TOOL FOR BREAST CANCER DETECTION AND CLASSIFICATION IN MRI . 2001 Proceedings of the 23rd Annual EMBS international Conference, October 25-28, Istanbul, Turkey,(2001).

[6] O.L. Mangasarian ,R.Setiono and W.H.Goldberg, "Pattern recognition via linear programming : theory and application to medical diagnosis ", in large scale numerical optimization ,T.F. Coleman and Y.Li, Eds ,pp. 22-31, SIAM ,(1990).

[7] T. Hothorn and B. Lausen, "Bagging tree classifiers for laser scanning images: A data- and simulation-based strategy,” Artif.Intell.Med., vol. 27, no. 1, pp. 65-79, Jan. (2003).

[8] Ishibuchi H., Nozaki K., and Tanaka H. "Distributed Representation of Fuzzy Rules and its Application to Pattern Classication”, Fuzzy Sets and Systems, vol. 52, pp. 21-32, (1992).

[9] R.Jang, “Anfis : adaptative network-based fuzzy inference système “,IEEE Trans.on Systems, Man and Cybernetics,J.S., (1993).

[10] R.R.Janghel, Anupam Shukla , Ritu Tiwari, Rahul Kala .”Breast Cancer Diagnosis using Artificial Neural Network Models", Information Sciences and Interaction Sciences (ICIS), 3rd International Conference on 2010, IEEE conferences , pp: 89 - 94, (2010).

[11] Kohonen T., "Self-organized Formatin of Topologically Correct Feature Maps", Bio. Cybem, vol. 43, pp. 59- 69 , (1982).

[12] Kohonen T. (1990), “The Self-organizing Map”, New Concepts in Computer Science: Proc. Symp. in Honour of Jean-Claude Simon, Pans, France, pp. 181-190, (1990).

[13] Ming $\mathrm{Li}$ and Zhi-Hua Zhou. "Improve Computer-Aided Diagnosis With Machine Learning Techniques Using Undiagnosed Samples. IEEE TRANSACTIONS ON SYSTEMS, MAN, AND CYBERNETICS-PART A /SYSTEM AND HUMAIN, VOL.37,NO .6 November, pp: 1088-1098, (2007).

[14] A.Lorenz, M.Blum, "Comparaison of different Neuro-Fuzzy Clasification Systems for the detection of Protstate Cancer in Ultrasonic Images". In Proc. of the IEEE 1997 Ultrasonics Symposium, pp. 1201-1204, (1997)

[15] P. Messad and G. Yen. "Combined Numerical and Linguistic Knowledge Representation and Its Application to Medical Diagnosis," in Component and Systems Diagnostics, Prognostics, and Health Management II, 2003.

[16] Mitra S., Pal S.K. "Self-organizing Neural Network as a Fuzzy Classifier", IEEE \%ns. Syst., Man and Cybern., vol. 24, no. 3, pp. 385-399 . (1994),

[17] Jyh-Shing Roger Jang ;Chuen-Tsai Sun; Eiji Mizutani ,'Neuro Fuzzy And Soft Computing”. A computational Approch to learning and Machine intelligence. Prentice Hall, (1997).

[18] Nadia Nedjah,Luiza de Macedo Mourelle . 'Fuzzy systems engineering: theory and practice" , éditeur :Springer, (2005).

[19] Asuncion, A \& Newman, D.J. "UCI Machine Learning Repository": http://www.ics.uci.edu/ mlearn/MLRepository.html. Irvine, CA: University of California, Department of Information and Computer Science , (2007). 
[20] Nozaki K., Ishibushi H, and Tanaka H. "Trainable Fuzzy Classification Systems Based on fizzy If-Then Rules", Proc. IEEE Int. Conf. on Fuzzy Systems, vol. 1,pp. 498-502, (1994),.

[21] Carlos Andrés Peña-Reyes and Moshe Sipper. "Designing breast cancer diagnosis systems via a hybrid fuzzy-genetic methodology”. January 7, (1999)

[22] Pena-Reyes, C.A. and M. Sipper (2000). "Applying Fuzzy CoCo to breast cancer diagnosis". Proceedings of the 2000 Congress on Evolutionary Computation, Volume: 2, pp. 1168 -1175, (2000).

[23] R. Setiono. "Extracting rules from pruned neural networks for breast cancer diagnosis". Artificial Intelligence in Medicine, pages 37-51, (1996).

[24] Mihir Sewak, Priyanka Vaidya, Chien-Chung Chan, Zhong-Hui Duan. "SVM Approach to Breast Cancer Classification". Second International Multisymposium on Computer and Computational Sciences . Pages :32-37 (2007).

[25] A. Sharkey, N. Sharkey, and S. Cross, "Adapting an ensemble approach for the diagnosis of breast cancer," in Proc.6th Int.Conf.Artif.Neural Netw., Skövd, Sweden, pp. 281-286,(1998).

[26] Pena-Reyes, C. A., \& Sipper, M. "A fuzzy-genetic approach to breast cancer diagnosis”, Artificial Intelligence in Medicine, Vol.17, pp:131-155,(1999).

[27] Y.Sun , F.Karray, S.Al- Sharhan . "Hybrid soft computing techniques for heterogenous data classification". Proceedings of the 2002 IEEE International Conference, Volume : 2 ,pp: 1511 1516,(2002) .

[28] Hee-Jun Song, Seon-Gu Lee, Gwi-Tae Park "A Methodology of Computer Aided Diagnostic System on Breast Cancer", Proceedings of the 2005 IEEE Conference on Control Applications Toronto, Canada, August 28-31, (2005).

[29] Pejman Tahmasebi, Ardeshir Hezarkhani ."Application of Adaptive Neuro-Fuzzy Inference System for Grade Estimation; Case Study, Sarcheshmeh Porphyry Copper Deposit", Kerman, Iran Department of Mining, Metallurgy and Petroleum Engineering, Amirkabir University, Hafez Ave. No. 424, Tehran, Iran. Australian Journal of Basic and Applied Sciences 2010 , INSInet Publication , (2010).

[30] Juan-Manuel TORRES-MORENO. "Apprentissage et généralisation par des réseaux de neurones : étude de nouveaux algorithmes constructifs ". Thèse pour obtenir le titre de docteur de l'institut national polytechnique de grenoble : Spécialité : Sciences Cognitives. France (1997).

[31] José António Barros Vieira, Fernando Morgado Dias, Alexandre Manuel Mota . "Comparison between artificial neural networks and neuro-fuzzy systems in modeling and control": a case study. IFAC. pp : 1-6, (2003)

[32] Jeen-Shing Wang and C. S. George Lee, "Self-Adaptive Neuro-Fuzzy Inference Systems for Classification Applications", IEEE Transactions on Fuzzy Systems, (2002).

[33] Street, W., Wolberg, W. and Mangasarian, O. "Machine Learning Techniques to Diagnose Breast Cancer from Image-Processed Nuclear Features of Fine Needle Aspirates", Cancer Letters Vol. 77 pages.163-171, (1994).

[34] Z.-H. Zhou, Y. Jiang, Y.-B. Yang, and S.-F. Chen, "Lung cancer cell identification based on artificial neural network ensembles,” Artif.Intell. Med., Vol. 24, No. 1, pp. 25-36, Jan. (2002).

\section{Authors}

Fatima Bekaddour received her Engineer degree in Computer Science Option Artifitial Intelligence (AI) from Tlemcen University, Algeria in 2010. She is a young researcher at the Biomedical Engineering Laboratory (TlemcenUniversity)And currently preparing her magister degree focusing on improving the performance of classifiers by using meta-heuristics. Her research interests have been in Soft Computing, optimization, computer aided medical decision support systems.

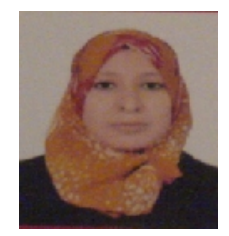

Mohamed Amine Chikh is graduated from The Electrical Engineering Institut (INELEC) Of Boumerdes - Algeria in 1985 with an Engineering degree in Computer science and in 1992 with a Magister of Electronic from Tlemcen University. He also received a Ph.D in electrical engineering from the University of Tlemcen (Algeria) and INSA of Rennes (France) in 2005. And is currently Professor at Tlemcen UniversityAlgeria. Actually he is the head of CREDOM research team at Biomedical Engineering Laboratory. He conducted post-doctoral teaching and research at the

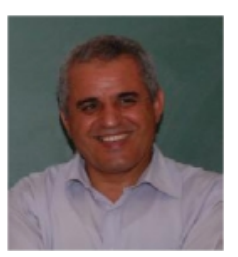
University of Tlemcen.Dr Chikh has published over 90 journal and conference papers to date and is involved in a variety of funded research projects related to biomedical engineering. He is a member of several scientific conferences. His research interests have been in artificial intelligence, machine learning, medical data classification, computer assisted medical decision support systems 